\title{
Albumin exfoliated titanium disulfide nanosheet: a multifunctional nanoplatform for synergistic photothermal/radiation colon cancer therapy
}

This article was published in the following Dove Press journal: OncoTargets and Therapy

\section{Chen Cao \\ Junhui Zhang \\ Chuanhua Yang \\ Lili Xiang \\ Wenneng Liu}

West China School of Public Health and West China Fourth Hospital, Sichuan University, Chengdu, Sichuan 6I004I, People's Republic of China
Correspondence: Wenneng Liu

No. 18, Section 3, Renmin South Road, Chengdu, Chengdu 610000, Sichuan,

People's Republic of China

Tel +86I 7780654303

Email liuwenneng@scu.edu.cn
Purpose: $\mathrm{TiS}_{2}$-HSA-FA, a nanoagent based on titanium disulfide $\left(\mathrm{TiS}_{2}\right)$, human serum albumin (HSA), and folic acid (FA), was synthesized for potential use in synergistic photothermal/radiation therapy for colon cancer.

Methods: $\mathrm{TiS}_{2}$ nanosheets were synthesized through a HSA-assisted exfoliation method and then modified with PEGylated FA. The morphology, size, zeta potential, stability, cellular uptake, cytotoxicity, biodistribution, and in vitro and in vivo biocompatibility of the nanoparticles as well as their suitability for synergistic photothermal/radiation colon cancer therapy were investigated.

Results: The as-synthesized $\mathrm{TiS}_{2}$-HSA-FA nanoparticles showed excellent physiological stability, as well as high absorption values in the near-infrared (NIR) and X-ray regions, giving them superb activity as a photothermal and radiation sensitizer. In vitro and in vivo experiments indicated that $\mathrm{TiS}_{2}$-HSA-FA showed high tumor targeting selectivity, blood circulation time, biocompatibility, and suitability for synergistic tumor photothermal radiotherapy.

Conclusion: A multifunctional nanoplatform based on $\mathrm{TiS}_{2}$ was developed and found to be potentially suitable for synergistic photothermal/radiation therapy for colon cancer.

Keywords: titanium disulfide, tumor target, photothermal therapy, radiotherapy, synergistic colon cancer therapy

\section{Introduction}

Although anti-tumor therapeutics have greatly improved in recent decades, malignant tumors are still a major threat to human beings' health worldwide. ${ }^{1,2}$ One of the most commonly used treatment strategies is radiotherapy (RT), in which tumors are exposed to ionizing radiation such as X-rays in an attempt to produce necrosis of tumor tissue through DNA damage and generation of reactive oxygen species. ${ }^{3-5}$ However, in practice, RT has several drawbacks. One is that it can harm normal tissue and thereby induce acute side effects such as nausea and vomiting, hair loss, etc., 6,7 Another is that considerable dose must be used to ensure that all tumor cells are killed, and yet X-ray irradiation has no tumor specificity. This necessitates excessively large radiation doses, inevitably causing injury to healthy tissues. To overcome these drawbacks, a series of radio-sensitizers such as the two-dimensional (2D) layered transition metal dichalcogenides (TMDs) $\left(\mathrm{MoS}_{2}, \mathrm{WS}_{2}, \mathrm{MoSe}_{2}\right.$, and $\left.\mathrm{ReS}_{2}\right)$ and other inorganic materials have been prepared. ${ }^{8-13}$ Most TMDs were reported to contain high- $Z$ elements that can act as radio-sensitizers to promote the cancer RT 
effect by absorbing X-rays and concentrating the radiation dose within tumors. ${ }^{14-16}$ For example, Shen and co-authors have prepared uniform ultrathin rhenium disulfide nanosheets for photothermal RT. ${ }^{16}$ However, the hypoxic environment inside tumors is still considered a major challenge for successful use of radio-sensitizers in RT. ${ }^{17,18}$

Photothermal therapy (PTT) has attracted substantial interest of late, as an alternative tumor treatment strategy. ${ }^{19,20}$ It is minimally invasive, and its high specificity means minimal side effects in normal tissues. PTT relies on photothermal agents to convert absorbed light energy into heat to kill tumor cells. $^{21-23}$ Recently, various nanomaterials such as organic small molecules, ${ }^{24,25}$ TMDs-based nanocomposites, ${ }^{19,26}$ protein-based nanoparticles, ${ }^{27,28}$ etc. have been developed as PTT nanoagents. $^{29-31}$ Among these, TMD-based nanoagents have demonstrated great potential for PTT because of their strong absorbance in the near IR (NIR) region and their large specific surface area which facilitates their functional modification. In addition, under NIR laser irradiation, TMD-based nanoagents can increase intratumor blood circulation, thereby facilitating oxygen availability inside the tumor, which could facilitate the RT effect. ${ }^{16,32,33}$ It is, therefore, thought that some type of TMD nanomaterial could be found which could act as an "all in one" nanoagent, perfectly combining RT and PTT for optimal therapeutic effect.

In this work, we prepared a new multifunctional nanoagent for combined PTT and RT, using tumor-targeted molecule FA and PEG modification of albumin exfoliated $\mathrm{TiS}_{2}$ nanosheets ( $\mathrm{TiS}_{2}-\mathrm{HSA}-\mathrm{FA}$ ). $\mathrm{TiS}_{2}$, as a new member of the TMD family, has been reported to have a high NIR absorbance which makes it an attractive candidate for PTT. ${ }^{34-36}$ Given its potential as a dual-modality therapy nanoagent, its efficacy as a radio-sensitizer is investigated here for the first time. The advantages of the developed nanoplatform $\mathrm{TiS}_{2}$-HSA-FA include: 1) improved stability and biocompatibility of $\mathrm{TiS}_{2}$ due to the PEG and HSA modification; 2) great radio-sensitivity; 3) high photothermal effect under NIR laser irradiation; and 4) remarkable tumor cell targeting effect after sequential conjugation with FA. The in vitro and in vivo results demonstrated that $\mathrm{TiS}_{2}$-HSA-FA are a highly effective nanoagent for synergistic photothermal/radiation therapy, which may ultimately prove to be suitable for clinical use.

\section{Methods}

\section{Materials}

Titanium disulfide $\left(\mathrm{TiS}_{2}\right.$, Crystal, $\left.99.995 \%\right)$ and Cell counting kit-8 (CCK-8) were purchased from Sigma-Aldrich (St. Louis,
Mo, USA). FA-PEG $5,000-\mathrm{NHS}$ and $\mathrm{CH}_{3}-\mathrm{PEG}_{5,000}-\mathrm{NHS}$ were obtained from $\mathrm{Xi}$ 'an Kaixin Biotech. Co. Ltd (China). Human serum albumin (HSA) and Fluorescein isothiocyanate (FITC) were obtained from Aladdin (Shanghai, China). Calcein-AM (CA)-propidium iodide (PI) stain were purchased from SigmaAldrich (St. Louis, Mo, USA).

\section{Preparation and characterizations of $\mathrm{Tis}_{2}-$ HSA-FA}

In detail, $20 \mathrm{mg}$ bulk $\mathrm{TiS}_{2}$ was added into $10 \mathrm{~mL}$ distilled water, following $2 \mathrm{hrs}$ ultrasonication ( $2 \mathrm{~s}$ on and $3 \mathrm{~s}$ off) in a sonicator Omni Sonic Ruptor 4000 (Omni International, Kennesaw, GA, USA). After that, $10 \mathrm{mg}$ HSA was added to the mixture and sonicated at the same condition for further $6 \mathrm{hrs}$. After centrifugation at $7000 \mathrm{rpm}$ for 18 mins, the supernatant was collected and centrifuged at $10,000 \mathrm{rpm}$ for $20 \mathrm{mins}$, resulting in $\mathrm{TiS}_{2}$ nanosheets

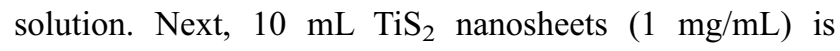
mixed with FA-PEG $5,000-\mathrm{NHS}(10 \mathrm{mg})$ and stirred for 2 hrs to be $\mathrm{TiS}_{2}$-HSA-FA. As a contrast, FA-PEG ${ }_{5,000}-\mathrm{NHS}$ was replaced by $\mathrm{CH}_{3}-\mathrm{PEG}_{5,000}-\mathrm{NHS}$ to form $\mathrm{TiS}_{2}-\mathrm{HSA}$. Lastly, the resulted mixture was purified by dialysis in distilled water over $24 \mathrm{hrs}$ to obtain $\mathrm{TiS}_{2}$-HSA and $\mathrm{TiS}_{2}$ HSA-FA. The morphology, size, zeta potential, XRD, and UV-VIS spectra of the resulted products were detected on atomic force microscopy (AFM, multimode, digital instruments, Veeco Company, USA), FEI transmission electron microscope (Eindhoven, The Netherlands), ZetaSizer Nano ZS (Malvern Instruments Ltd., UK), X-ray diffractometer (D8 ADVANCE, Bruker, Germany), UV-VIS spectrophotometry (UV-Vis 1800, Shimadzu, Kyoto, Japan), Fourier transform infrared spectroscopy (FT-IR, Bruker Optics GmbH, Ettlingen, Germany), respectively.

\section{Cell culture}

CT26 cells (mice colon cancer cell line) were obtained from Type Culture Collection of Chinese Academy of Science (Shanghai, China). CT26 cells were cultured in RPMI 1640 medium supplemented with 10\% fetal bovine serum, $1 \%$ penicillin and streptomycin in an atmosphere of $5 \% \mathrm{CO}_{2}$ at $37^{\circ} \mathrm{C}$.

\section{Cellular uptake and in vitro biocompatibility}

According to previous literatures, a classic small molecule dye, FITC, was applied to label the nanoparticles by simple physical absorption. After $24 \mathrm{hrs}$ incubating, CT26 cells were cultured with free FITC, TiS $_{2}-\mathrm{HSA}, \mathrm{TiS}_{2}-\mathrm{HSA}-\mathrm{FA}+\mathrm{FA}$, and 
$\mathrm{TiS}_{2}$-HSA-FA for $4 \mathrm{hrs}$, respectively. And then, the treated cells were successively washed by phosphate buffer saline (PBS) for three time slightly and fixed by glutaraldehyde, stained with DAPI for 10 mins. A commercial confocal laser scanning microscope was used to observe the fluorescence signals inside cells. Moreover, the treated cells were homogenized, and treated with $1 \mathrm{~mL}$ aqua regia solution for $5 \mathrm{hrs}$. An inductively coupled plasma-atomic emission spectrometry (ICP-AES, Hitachi P4010, Japan) was applied to detect the Ti content in the cells.

The in vitro biocompatibility of $\mathrm{TiS}_{2}-\mathrm{HSA}$ and $\mathrm{TiS}_{2}-$ HSA-FA on cells was evaluated by a standard CCK-8 assay. In detail, CT26 cells $\left(1 \times 10^{5}\right.$ cells $\left./ \mathrm{mL}, 0.5 \mathrm{~mL}\right)$ were cultured in 96-well plate for $24 \mathrm{hrs}$ and then, the cells were treated with different concentration of $\mathrm{TiS}_{2}$ HSA and $\mathrm{TiS}_{2}$-HSA-FA. After $24 \mathrm{hrs}$ incubation, the cell viability was detected by CCK- 8 assay.

\section{In vitro synergistic PTT/RT}

CT26 cells were treated with different concentration of $\mathrm{TiS}_{2}$-HSA and $\mathrm{TiS}_{2}$-HSA-FA $(0,10,30,50,100,150 \mu \mathrm{g} /$ $\mathrm{mL}$ ) and were cultured in $37^{\circ} \mathrm{C}$ incubator for $5 \mathrm{hrs}$. After washing the nanoparticles outside the cells, they were irradiated with NIR irradiation $(808 \mathrm{~nm}$ laser at a power density of $0.8 \mathrm{~W} / \mathrm{cm}^{2}$ for 5 mins) or RT (5Gy), or their combination. And then, the treated cells were further cultured for $24 \mathrm{hrs}$. During the 5 mins NIR irradiation, a handheld thermal camera (Ti27, Fluke, USA) was used to record the temperature change of the cells. The cell death rate was calculated using a typical CCK-8 assay.

As above, the cells were treated with $\mathrm{TiS}_{2}-\mathrm{HSA}$ and $\mathrm{TiS}_{2-}$ HSA-FA, following by irradiation with NIR irradiation and Xray at the same condition. According to the previous literatures, the cells were further stained with calcein-AM/PI and $\gamma-\mathrm{H} 2 \mathrm{AX}$, respectively, to evaluate the cell death. The fluorescence signal of stained cells was observed by a confocal laser scanning microscope (LSM 510 NLO META, Zeiss, Germany).

\section{Animal model}

We have revised the ethics statement: All animal experiments were strictly complied with the guideline of Animal Protection and Care Committee of Sichuan University. All animal experimental procedures involved in this work were approved by the Ethics Committee of Sichuan University. For CT26 tumor xenograft models, $10^{6}$ CT26 cells in $150 \mu \mathrm{L}$ PBS were subcutaneously injected into the right back of mice. After 7-10 days of inoculation, the tumor size was detected by a caliper.

\section{In vivo synergistic PTT/RT}

To investigate the synergistic PTT/RT, tumor-bearing mice were randomly divided into 5 groups $(\mathrm{n}=5)$ : group 1 : control $+\mathrm{NIR}+\mathrm{RT}$; group 2: $\mathrm{TiS}_{2}$-HSA-FA + RT; group 3: $\mathrm{TiS}_{2-}$ HSA-FA + NIR; group 4: TiS $_{2}$-HAS + RT+NIR; and group 5: $\mathrm{TiS}_{2}$-HSA-FA + RT+NIR (with $6 \mathrm{mg} / \mathrm{kg}$ of TiS ${ }_{2}$ ). NIR irradiation used $808 \mathrm{~nm}$ wavelength laser with $0.8 \mathrm{~W} / \mathrm{cm}^{2}$. RT applied 5 Gy X-ray for 5 mins. The temperature change of the tumor region when irradiated by NIR was recorded using an infrared thermal camera. Every three days treatment, the tumor size was recorded and the tumor volume was calculated according to the equation: $\mathrm{V}=$ length $\times$ width $^{2} / 2$. The relative tumor volume was calculated as $V / V_{0}\left(V\right.$ : current volume, $V_{0}$ : initial tumor volume). After the whole treatment, the major organs of these mice were collected for hematoxylin and eosin (H\&E) staining. In addition, the blood was collected and the index including white blood cell (WBC), red blood cells (RBC), hemoglobin (HGB), mean corpuscular hemoglobin $(\mathrm{MCH})$, hematocrit (HCT), mean corpuscular hemoglobin concentration (MCHC), mean corpuscular volume (MCV), and platelet (PLT) were detected.

\section{Statistical analysis}

All data were presented as mean with standard deviation (SD). Statistical analysis was analyzed with OriginPro 2016 via Students's $t$-test. The differences were considered significant for $* P<0.05$ and highly significant for $* * P<0.01$.

\section{Results and discussion Preparation and characterization of $\mathrm{TiS}_{2-}$ HSA-Fa}

$\mathrm{TiS}_{2}$ nanosheets were first prepared by HSA-assisted ultrasonication, and their sheet structure was confirmed by AFM image (Figure S1). They were then modified by PEGylated FA to produce $\mathrm{TiS}_{2}$-HSA-FA. TEM imaging shows that $\mathrm{TiS}_{2-}$ HSA-FA has a sheet-like structure with a lattice plane spacing of $0.24 \mathrm{~nm}$ (Figure 1A). As shown in Figure 1B, the sharp and narrow XRD peaks in $\mathrm{TiS}_{2}$-HSA-FA indicate good crystallinity of the prepared $\mathrm{TiS}_{2}$-HSA-FA, consistent with observations from HRTEM (Figure 1A, inset). In addition, both bulk $\mathrm{TiS}_{2}$ and $\mathrm{TiS}_{2}$-HSA-FA showed the same phase structure, demonstrating that the exfoliation process has no significant influence on the crystalline structure of the material. ${ }^{37}$ According to DLS analysis, the zeta potential and average diameter of $\mathrm{TiS}_{2}$-HSA-FA nanoparticles were approximately $-31.2 \mathrm{mV}$ and $135.3 \mathrm{~nm}$, respectively (Figure 1C and D). After 15 days of storage in physiological solution, the size of the 
A
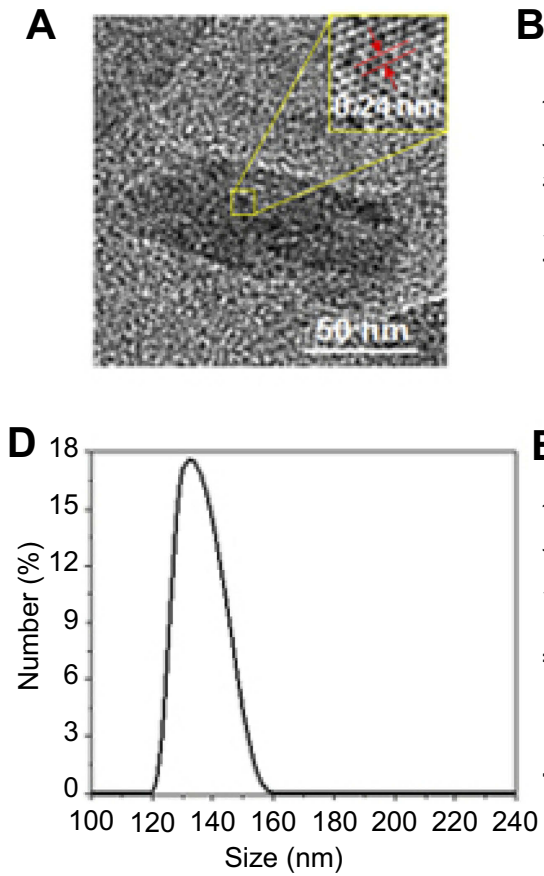

B

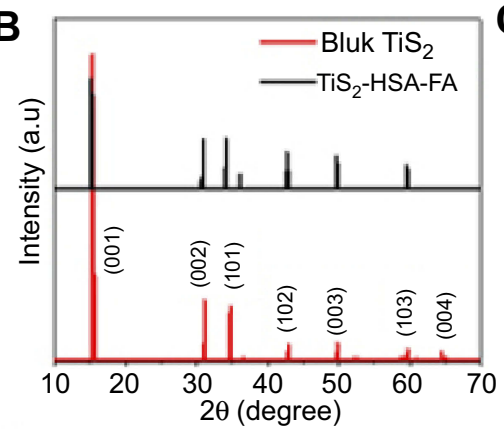

E

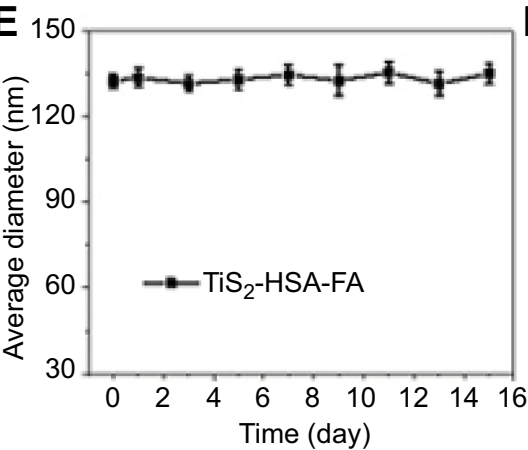

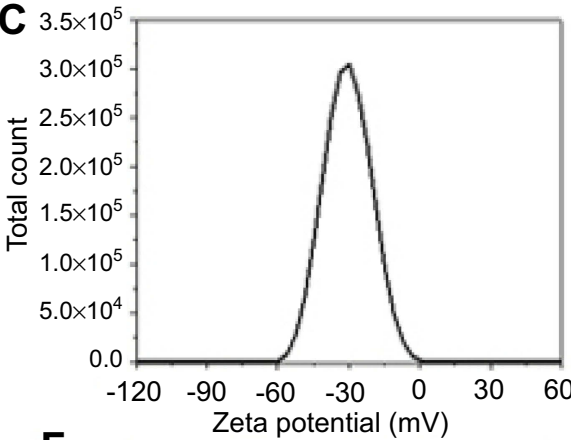

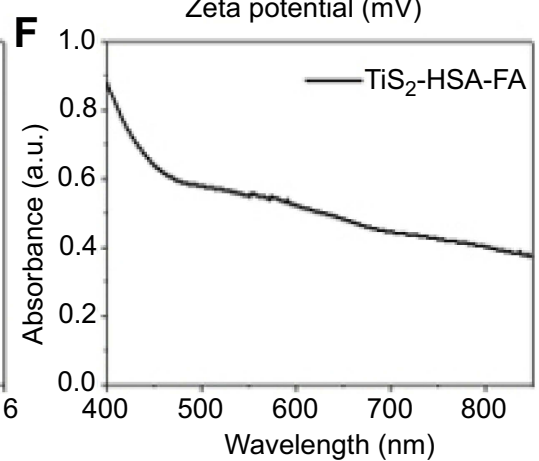

Figure I Preparation and characterization.

Notes: (A) TEM image of TiS 2 -HSA-FA. Inset was the high-resolution TEM image. (B) XRD pattern of bulk TiS ${ }_{2}$ and TiS $-\mathrm{HSA}-\mathrm{FA}$. (C) The zeta potential distribution of TiS $-\mathrm{HSA}-$ FA. (D) The size distribution of $\mathrm{TiS}_{2}-\mathrm{HSA}-\mathrm{FA}$. (E) The size change of $\mathrm{TiS}_{2}-\mathrm{HSA}-\mathrm{FA}$ in physiological solution over 15 days. (F) The absorbance spectra of TiS ${ }_{2}-\mathrm{HSA}-\mathrm{FA}$.

Abbreviations: $\mathrm{TiS}_{2}$, titanium disulfide; HSA, human serum albumin; FA, folic acid; TEM, transmission electron microscopy; XRD, X-ray diffraction.

$\mathrm{TiS}_{2}$-HSA-FA nanoparticles displayed no notable changes (Figure 1E). This stability is likely due to the absorption of HSA onto the surface of the particles, and subsequent modification with PEG. The UV-Vis spectrum of $\mathrm{TiS}_{2}$-HSA-FA in Figure $1 \mathrm{~F}$ shows high absorbance across all wavelengths from UV to NIR. The extinction coefficient of $\mathrm{TiS}_{2}$-HSA-FA at 808 $\mathrm{nm}$ was found to be $30.1 \mathrm{~L} / \mathrm{g} \mathrm{cm}$. In addition, as shown in Figure S2, the more intense $\sim 1650 \mathrm{~cm}^{-1}$ peak in $\mathrm{TiS}_{2}$-HSA-FA indicated the existence of the amido bond between $\mathrm{TiS}_{2}-\mathrm{HSA}$ and FA.

\section{Photothermal effect of $\mathrm{TiS}_{2}-\mathrm{HSA}-\mathrm{FA}$}

To test the photothermal properties of $\mathrm{TiS}_{2}$-HSA-FA, different concentrations $(0,50,100$, and $150 \mathrm{mg} / \mathrm{mL})$ of the nanoparticles were irradiated by NIR laser $(808 \mathrm{~nm}$, $0.8 \mathrm{~W} / \mathrm{cm}$ ) for 5 mins. The results showed that laser irradiation produced significant concentration-dependent temperature increases in $\mathrm{TiS}_{2}$-HSA-FA (Figure 2A). The highest temperature that could be reached was $65.3^{\circ} \mathrm{C}$. According to previous literatures, ${ }^{38-40}$ the photothermal conversion efficiency of $\mathrm{TiS}_{2}$-HSA-FA was calculated to be $\sim 58.9 \%$. The photothermal stability of $\mathrm{TiS}_{2}$-HSA-FA was also investigated (Figure $2 \mathrm{~B}$ ); $\mathrm{TiS}_{2}$-HSA-FA were clearly very stable throughout five cycles of NIR irradiation. In addition, the Hounsfield unit values of $\mathrm{TiS}_{2}$-HSA-FA, detected by computed tomography, were positively correlated with concentration (Figure 2C), demonstrating that $\mathrm{TiS}_{2}$-HSA-FA is a very effective radio-sensitizer.

\section{Cellular uptake and in vitro biocompatibility}

After labeling by FITC, the behavior of $\mathrm{TiS}_{2}-\mathrm{HSA}$ and $\mathrm{TiS}_{2}$-HSA-FA in cells was tracked by fluorescence microscopy. The fluorescence images showed that cells treated with $\mathrm{TiS}_{2}$-HSA-FA showed much more fluorescence signal in cytoplasm than those treated with $\mathrm{TiS}_{2}$-HSA-FA + FA, TiS $_{2}$-HSA, and free FITC (Figure 3A). ICP-AES quantitative analysis confirmed these results (Figure 3B). This indicates that FA conjugation significantly accelerated endocytosis of $\mathrm{TiS}_{2-}$ HSA-FA. In addition, the in vitro biocompatibility of the $\mathrm{TiS}_{2}$-HSA-FA was evaluated by standard CCK-8 assay. As shown in Figure 3C, no significant cytotoxicity of $\mathrm{TiS}_{2}$-HSA-FA was found even at high concentrations up to $0.8 \mathrm{mg} / \mathrm{mL}$, indicating good in vitro biocompatibility for both $\mathrm{TiS}_{2}$-HSA and $\mathrm{TiS}_{2}$-HSA-FA. 

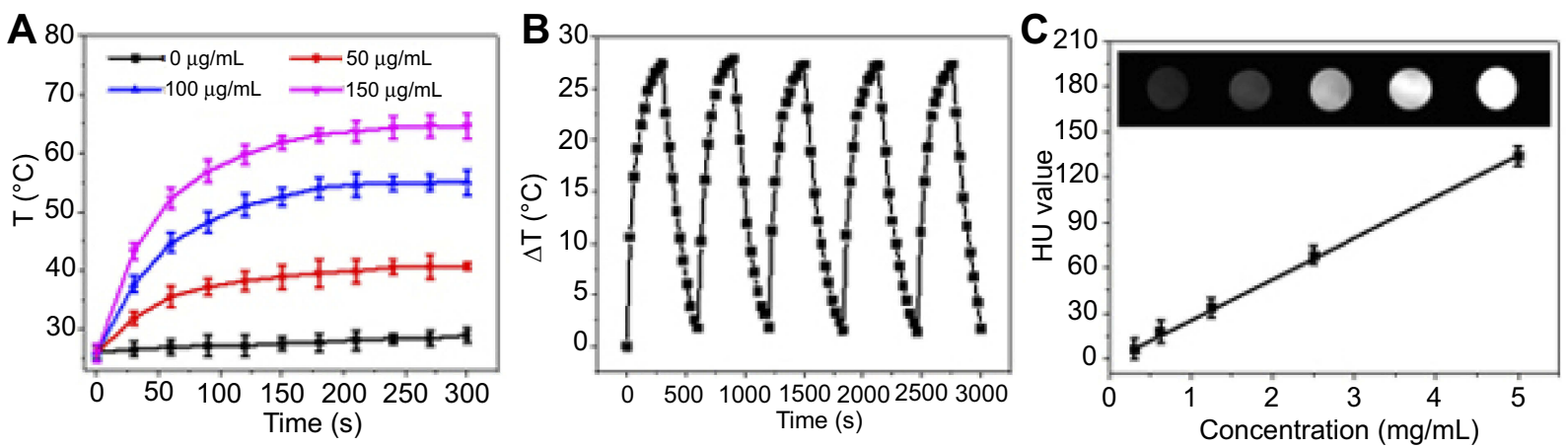

Figure 2 The photothermal and radio-sensitization property.

Notes: $(\mathbf{A})$ Temperature changes of $\mathrm{TiS}_{2}$-HSA-FA solution with various concentrations under $808 \mathrm{~nm}$ laser irradiation $\left(0.8 \mathrm{~W} / \mathrm{cm}^{2}\right)$. $(\mathbf{B})$ Temperature variations of TiS ${ }_{2}-\mathrm{HSA}-\mathrm{FA}$ under 5 cycles of $808 \mathrm{~nm}$ laser irradiation $\left(0.8 \mathrm{~W} / \mathrm{cm}^{2}\right)$. (C) The correlation between $\mathrm{HU}$ values and different concentrations of TiS $-\mathrm{HSA}-\mathrm{FA}$. The inset is CT images.

Abbreviations: $\mathrm{TiS}_{2}$, titanium disulfide; HSA, human serum albumin; FA, folic acid; CT, computed tomography.

A

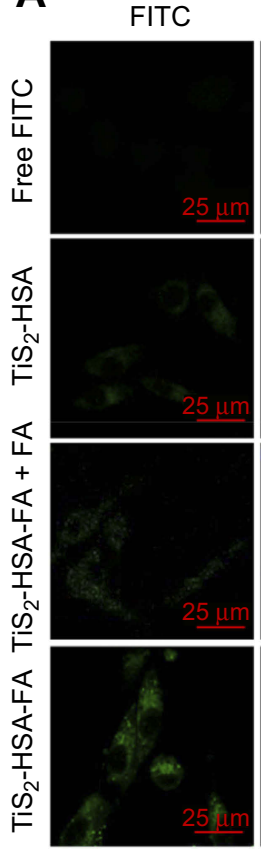

DAPI
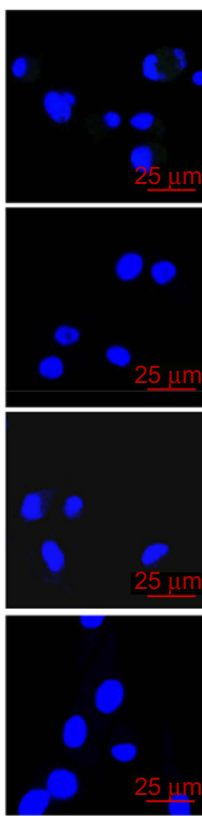

Overlay

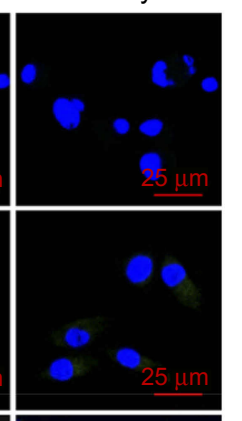

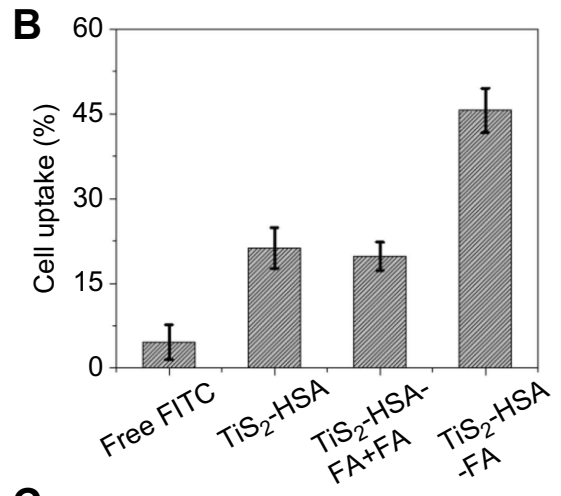

C
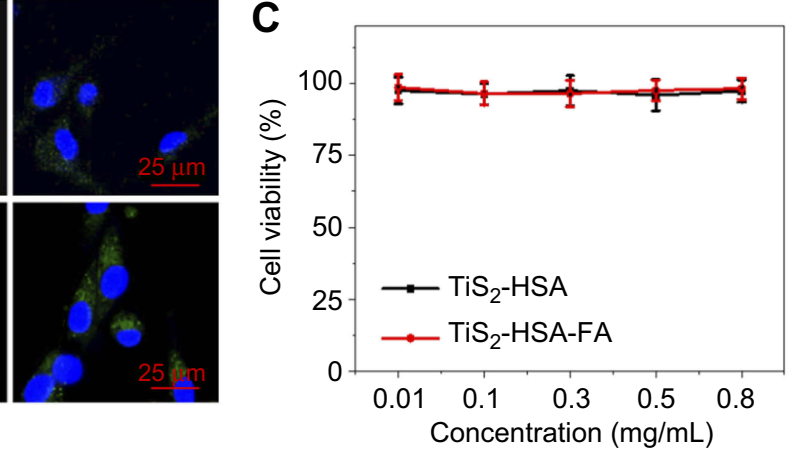

Figure 3 Cell uptake and biocompatibility.

Notes: (A) Fluorescence images of CT26 cells incubated with free FITC and FITC labeled TiS ${ }_{2}-\mathrm{HSA}, \mathrm{TiS}_{2}-\mathrm{HSA}-\mathrm{FA}+\mathrm{FA}$ blocking, and TiS 2 -HSA-FA. (B) Quantitative analysis of CT26 cells towards free FITC and FITC labeled TiS $\mathrm{S}_{2}-\mathrm{HSA}$, TiS 2 -HSA-FA + FA blocking, and TiS $-\mathrm{HSA}-\mathrm{FA}$ by ICP-AES. (C) Cell viability of CT26 cells after 24 hrs treatment with different concentrations of $\mathrm{TiS}_{2}-\mathrm{HSA}$ and $\mathrm{TiS}_{2}-\mathrm{HSA}-\mathrm{FA}$.

Abbreviations: $\mathrm{TiS}_{2}$, titanium disulfide; HSA, human serum albumin; FA, folic acid; ICP-AES, inductively coupled plasma-atomic emission spectrometry; FITC, fluorescein isothiocyanate; DAPI, 4',6-diamidino-2-phenylindole.

\section{In vitro synergistic PTT/RT}

As shown in Figure 4A, CT26 cells treated with $\mathrm{TiS}_{2}$-HSA-FA showed $28.5^{\circ} \mathrm{C}$ of temperature increase after 5 mins of NIR laser irradiation $\left(808 \mathrm{~nm}, 0.8 \mathrm{~W} / \mathrm{cm}^{2}\right)$, which was higher than that of either $\mathrm{TiS}_{2}$-HSA-treated or control-treated cells. Since the data from Figure 2C suggested that $\mathrm{TiS}_{2}$ has potential as a radiosensitizer, the in vitro RT efficacy of $\mathrm{TiS}_{2}$-HSA-FA was tested: as seen in Figure 4B, cell viability decreased with increasing Xray dose. $\mathrm{TiS}_{2}$-HSA-FA + RT exhibited greater inhibition of cell viability than either $\mathrm{TiS}_{2}$-HSA-treated or control-treated cells. This is likely because $\mathrm{TiS}_{2}$-HSA-FA is more effectively endocytosed, and thus can absorb the energy of X-ray radiation more effectively inside tumor cells and channel it into the formation of secondary Auger electrons to damage DNA. ${ }^{41-43}$ 

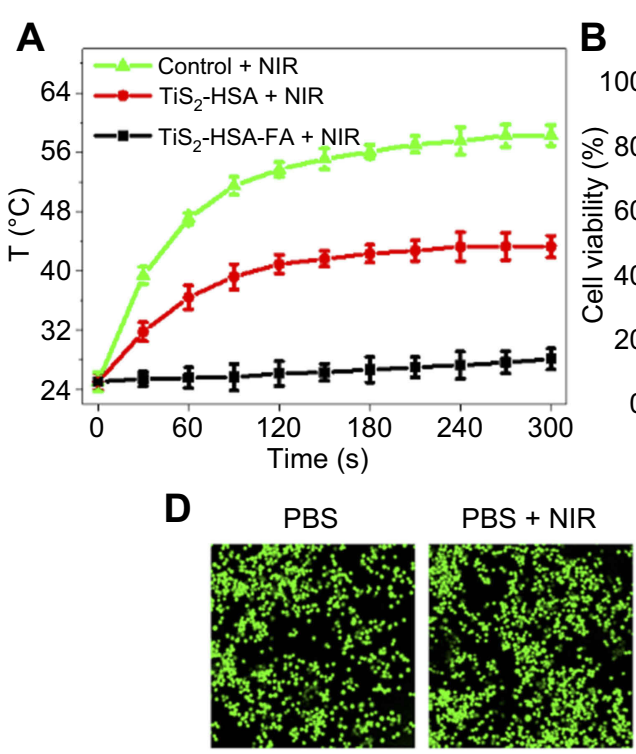

$\mathrm{TiS}_{2}$-HSA-FA $\mathrm{TiS}_{2}$-HSA-FA + NIR
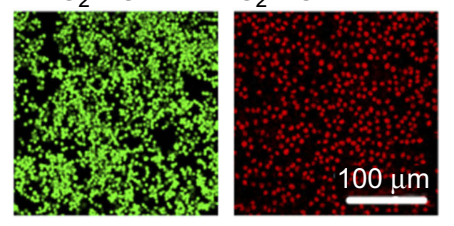
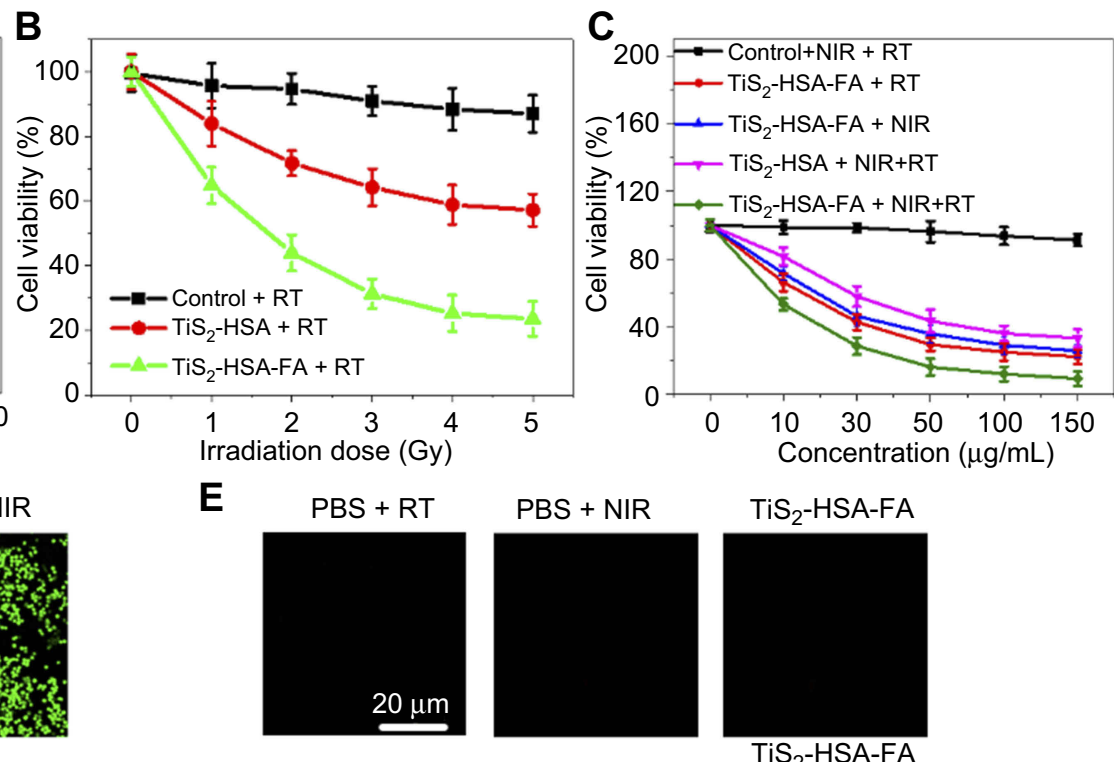

$\mathrm{TiS}_{2}$-HSA-FA + RT $\mathrm{TiS}_{2}-\mathrm{HSA}-\mathrm{FA}+\mathrm{NIR}+\mathrm{NIR}+\mathrm{RT}$
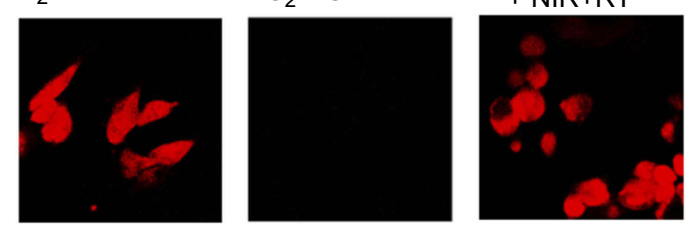

Figure 4 In vitro photothermal radiotherapy.

Notes: (A) Temperature change curve of control, TiS $2-\mathrm{HSA}$ and TiS $2-\mathrm{HSA}-\mathrm{FA}$ under 5 mins NIR irradiation $\left(808 \mathrm{~nm}, 0.8 \mathrm{~W} / \mathrm{cm}^{2}\right)$. (B) Cell viability of CT26 cells treated by $\mathrm{TiS}_{2}-\mathrm{HSA}$ and $\mathrm{TiS}_{2}-\mathrm{HSA}-\mathrm{FA}$ and were irradiated by different dose of X-ray. (C) Cell viability of CT26 cells treated by $\mathrm{TiS}_{2}-\mathrm{HSA}$ and TiS 2 -HSA-FA and irradiated by NIR laser or X-ray. (D) Live-dead staining images of CT26 cells treated with PBS, PBS + NIR, TiS 2 -HSA-FA, TiS 2 -HSA-FA + NIR. (E) $\gamma$-H2AX staining images of CT26 cells treated with PBS + RT, PBS + NIR, TiS - HSA-FA, TiS $2-H S A-F A+N I R, ~ T i S_{2}-\mathrm{HSA}-\mathrm{FA}+\mathrm{RT}$, and $\mathrm{TiS}_{2}-\mathrm{HSA}-\mathrm{FA}+\mathrm{NIR}+\mathrm{RT}$, respectively.

Abbreviations: $\mathrm{TiS}_{2}$, titanium disulfide; HSA, human serum albumin; FA, folic acid; RT, radiation therapy; NIR, near infrared; PBS, phosphate buffer saline.

The effect of combined PTT/RT treatment was then investigated. Cells were treated with control $+\mathrm{NIR}+\mathrm{RT}$, $\mathrm{TiS}_{2}$-HSA-FA + RT, TiS 2 -HSA-FA + NIR, TiS $_{2}$-HAS + NIR + RT, or TiS 2 -HSA-FA + NIR + RT. As shown from the standard CCK-8 assay, under NIR and RT treatment, TiS $_{2}$-HSA-FA displayed the highest cell inhibition rate (93.4\%) of all the groups (Figure 4C).

Calcein-AM/PI staining and $\gamma$-H2AX immunofluorescence were further used to evaluate the combined effect of PTT and RT. The RT- and PTT-treated cells were stained by calcein-AM/ PI. As shown in Figure 4D, almost all the cells were killed in the $\mathrm{TiS}_{2}$-HSA-FA + NIR + RT group, while few were killed in the PBS, PBS + NIR, and $\mathrm{TiS}_{2}$-HSA-FA groups. Figure 4E shows the results of $\gamma$-H2AX immunofluorescence. In PBS + RT, PBS + NIR, TiS $_{2}$-HSA-FA, and TiS $_{2}$-HSA-FA + NIR-treated groups, negligible $\gamma-\mathrm{H} 2 \mathrm{AX}$ immunofluorescence was seen. By contrast, the $\mathrm{TiS}_{2}$-HSA-FA + RT and $\mathrm{TiS}_{2}$-HSA-FA + NIR + RT-treated groups exhibited strong $\gamma-\mathrm{H} 2 \mathrm{AX}$ immunofluorescence signals, suggesting that $\mathrm{TiS}_{2}$-HSA-FA can enhance the radio-sensitivity of cells and produce more DNA damage.

\section{In vivo biodistribution and blood circulation}

Titanium content of the major organs post-injection of $\mathrm{TiS}_{2^{-}}$ HSA and $\mathrm{TiS}_{2}$-HSA-FA were evaluated. The majority of the two nanoparticles were distributed in liver and kidney. Much more $\mathrm{TiS}_{2}$-HSA-FA was collected in tumor tissue than $\mathrm{TiS}_{2}$-HSA (Figure $5 \mathrm{~A}$ ). The concentration of $\mathrm{TiS}_{2}$ HSA-FA in tumor tissue peaked at $24 \mathrm{hrs}$ post-injection (Figure 5B). The accumulation of $\mathrm{TiS}_{2}$-HSA-FA in tumor was likely due to the FA modification, which is known to facilitate tumor targeting. ${ }^{44,45}$ As shown in Figure 5C, after intravenous injection, blood distribution half-life $\left(\mathrm{t}_{1 / 2} \alpha\right)$ and blood terminal elimination half-life $\left(\mathrm{t}_{1 / 2} \beta\right)$ of the $\mathrm{TiS}_{2}$-HSAFA were $1.21 \pm 0.11 \mathrm{hrs}$ and $17.52 \pm 0.62 \mathrm{hrs}$, respectively. It has been reported that PEG and HSA modifications can prolong blood circulation and reduce macrophage clearance of nanoparticles from the reticuloendothelial system. ${ }^{46,47}$

\section{In vivo synergistic PTT/RT}

For in vivo synergistic PTT/RT, NIR (5 mins, $808 \mathrm{~nm}$, $0.8 \mathrm{~W} / \mathrm{cm}^{2}$ ) and X-ray (5 mins, 5Gy) irradiation were applied 

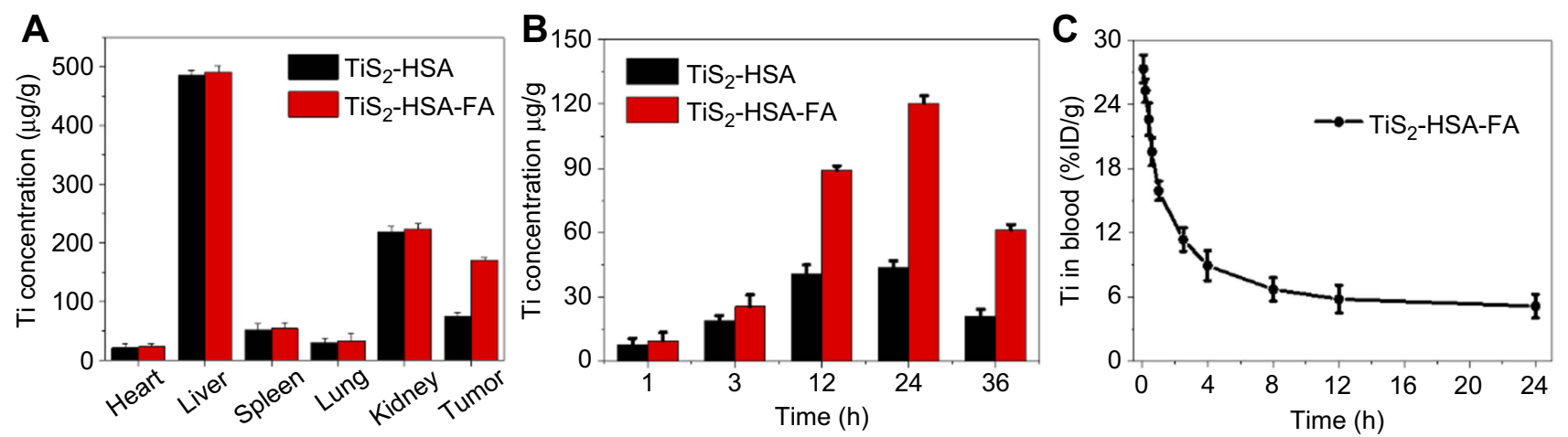

Figure 5 In vivo biodistribution and blood circulation.

Notes: (A) Ti concentration in tumor and major organs (heart, liver, spleen, lung, and kidney) of $\mathrm{TiS}_{2}$-HSA- and TiS $-\mathrm{HSA}-\mathrm{FA}$-treated mice. (B) Ti concentration in tumor region of $\mathrm{TiS}_{2}-\mathrm{HSA}$ - and $\mathrm{TiS}_{2}-\mathrm{HSA}-\mathrm{FA}$-treated mice at different time points. (C) Ti concentration in blood at different time points after injection of TiS $\mathrm{S}_{2} \mathrm{HSA}-\mathrm{FA}$.

Abbreviations: $\mathrm{TiS}_{2}$, titanium disulfide; HSA, human serum albumin; FA, folic acid.

$24 \mathrm{hrs}$ after intravenous injection. The temperature of the tumor region was recorded by a thermal imager. Figure $6 \mathrm{~A}$ and $\mathrm{B}$ showed a roughly $25^{\circ} \mathrm{C}$ increase in $\mathrm{TiS}_{2}$-HSA-FA + NIR-treated mice, compared to those treated with control or $\mathrm{TiS}_{2}$-HAS + NIR. The weight of the mice increased normally over the duration of the 21-day treatment (Figure 7A). The best tumor growth inhibition effect was observed with combined PTT/RT in group $5\left(\right.$ TiS $_{2}$-HSA-FA + NIR+RT). The results demonstrated that PTT/RT delivered by $\mathrm{TiS}_{2}$-HSA-FA have an obviously synergistic therapeutic outcome compared to PTT or RT alone (Figure 7B).

After the treatment, the heart, liver, spleen, lung, kidney, and whole blood were collected for HE section staining and complete blood panel assays to evaluate the biocompatibility of the nanoparticles. As seen in Figure 8A and B, no obvious differences were observed between the $\mathrm{TiS}_{2}$-HSAFA group and the control group. This indicates that $\mathrm{TiS}_{2}-$ HSA-FA has good in vivo biocompatibility.

\section{Conclusion}

In summary, we have prepared a biocompatible PTT/RT nanoplatform $\mathrm{TiS}_{2}$-HSA-FA by using simple ultrasonication to create albumin exfoliated $\mathrm{TiS}_{2}$ nanosheets that were then modified with PEG and FA. The TiS 2 -HSA-FA nanoparticles showed strong NIR light absorbance, good stability, and remarkable efficacy as a radio-sensitizer. In vitro and in vivo characterization showed $\mathrm{TiS}_{2}$-HSA-FA to have a high specificity for targeting tumors, likely due to its modification with FA. The nanoparticles also produced a remarkable, synergistic, cytotoxic effect under PTT/RT irradiation, and showed great biocompatibility in vitro and in vivo. This multifunctional nanoplatform shows great promise for future use as a new tumor therapeutic agent.

\section{Disclosure}

The authors report no conflicts of interest in this work.


Figure 6 In vivo photothermal performance.

Notes: (A) In vivo thermal images of mice after intravenous injection of saline, $\mathrm{TiS}_{2}-\mathrm{HSA}$ and TiS 2 -HSA-FA under 5 mins NIR irradiation $\left(808 \mathrm{~nm}, 0.8 \mathrm{~W} / \mathrm{cm}^{2}\right.$ ). (B) The corresponding temperature change curves of tumor regions in mice.

Abbreviations: $\mathrm{TiS}_{2}$, titanium disulfide; HSA, human serum albumin; FA, folic acid; RT, radiation therapy; NIR, near infrared. 

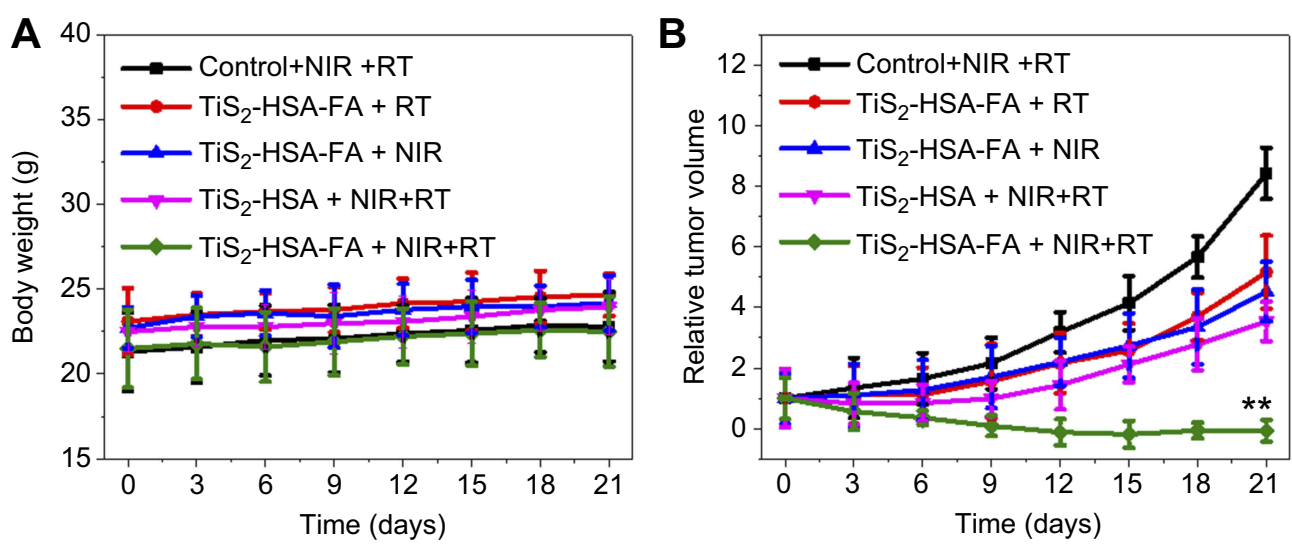

Figure 7 In vivo synergistic photothermal radiotherapy.

Notes: The change of $(\mathbf{A})$ body weight and $(\mathbf{B})$ relative tumor volume of CT26-bearing mice in different groups, including control (saline) + NIR+RT, TiS 2 -HSA-FA + RT, $\mathrm{TiS}_{2}-\mathrm{HSA}-\mathrm{FA}+\mathrm{NIR}, \mathrm{TiS}_{2}-\mathrm{HAS}+\mathrm{NIR}+\mathrm{RT}$, and $\mathrm{TiS}_{2}-\mathrm{HSA}-\mathrm{FA}+\mathrm{NIR}+\mathrm{RT}$. $* * \mathrm{P}<0.01$, compared to other groups.

Abbreviations: $\mathrm{TiS}_{2}$, titanium disulfide; HSA, human serum albumin; FA, folic acid; RT, radiation therapy; NIR, near infrared.

A

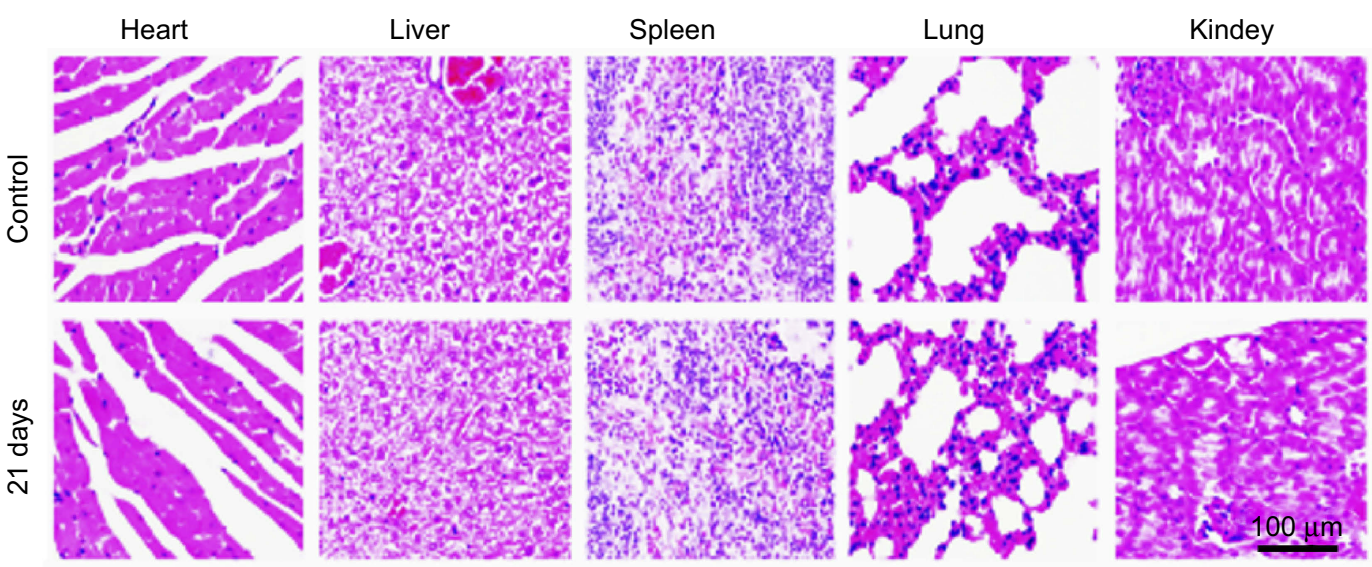

B

\begin{tabular}{|c|c|c|c|c|}
\hline $\begin{array}{c}\text { Normal } \\
\text { range }\end{array}$ & $5.29-15.21$ & $\begin{array}{c}\mathrm{RBC} \\
\left(10^{12} / \mathrm{L}\right)\end{array}$ & $\mathrm{HGB}(\mathrm{g} / \mathrm{L})$ & $\mathrm{MCH}(\mathrm{pg})$ \\
\hline Control & $13.4 \pm 0.38$ & $9.56 \pm 0.78$ & $165.3 \pm 11.1$ & $16.8 \pm 0.8$ \\
\hline 21 days & $12.5 \pm 0.71$ & $8.96 \pm 0.66$ & $171.25 \pm 9.5$ & $17.9 \pm 0.7$ \\
\hline $\begin{array}{c}\text { Normal } \\
\text { range }\end{array}$ & $41-71$ & $242-324$ & $50.1-64.9$ & $471-1598$ \\
\hline Control & $61.3 \pm 1.1$ & $295.6 \pm 8.5$ & $60.2 \pm 0.8$ & $1325.1 \pm 71$ \\
\hline 21 days & $56.5 \pm 1.4$ & $308.1 \pm 9.3$ & $58.1 \pm 0.64$ & $1246.9 \pm 50$ \\
\hline
\end{tabular}

Figure 8 In vivo biocompatibility.

Notes: (A) HE-stained images of heart, liver, spleen, lung, and kidney collected from mice after $2 \mathrm{I}$ days treatment (scale bar $=100 \mu \mathrm{m})$. $(\mathbf{B})$ Blood biochemistry of mice after 21 days treatment.

Abbreviations: $\mathrm{TiS}_{2}$, titanium disulfide; HSA, human serum albumin; FA, folic acid; HE, hematoxylin and eosin; WBC, white blood cell; RBC, red blood cells; HGB, hemoglobin; $\mathrm{MCH}$, mean corpuscular hemoglobin; HCT, hematocrit; MCHC, mean corpuscular hemoglobin concentration; MCV, mean corpuscular volume; PLT, platelet. 


\section{References}

1. Fitzmaurice C, Dicker D, Pain A, et al. The global burden of cancer 2013. JAMA Oncol. 2015;1(4):505-527. doi:10.1001/jamaoncol.2015.0735

2. Cvek B. Nonprofit drugs as the salvation of the world's healthcare systems: the case of Antabuse (disulfiram). Drug Discov Today. 2012;17(9-10):409-412. doi:10.1016/j.drudis.2011.12.010

3. Siva S, MacManus MP, Martin RF, Martin OA. Abscopal effects of radiation therapy: a clinical review for the radiobiologist. Cancer Lett. 2015;356(1):82-90.

4. Bentzen SM. Preventing or reducing late side effects of radiation therapy: radiobiology meets molecular pathology. Nat Rev Cancer. 2006;6(9):702.

5. Prise KM, Schettino G, Folkard M, Held KD. New insights on cell death from radiation exposure. Lancet Oncol. 2005;6(7):520-528.

6. Santivasi WL, Xia F. Ionizing radiation-induced DNA damage, response, and repair. Antioxid Redox Signal. 2014;21(2):251-259.

7. Patyar RR, Patyar S. Role of drugs in the prevention and amelioration of radiation induced toxic effects. Eur J Pharmacol. 2018;819:207-216.

8. Ma N, Jiang YW, Zhang X, et al. Enhanced radiosensitization of gold nanospikes via hyperthermia in combined cancer radiation and photothermal therapy. ACS Appl Mater Interfaces. 2016;8(42):28480-28494.

9. Ma N, Wu FG, Zhang X, et al. Shape-dependent radiosensitization effect of gold nanostructures in cancer radiotherapy: comparison of gold nanoparticles, nanospikes, and nanorods. ACS Appl Mater Interfaces. 2017;9(15):13037-13048.

10. Jiang YW, Gao G, Jia HR, et al. Copper oxide nanoparticles induce enhanced radiosensitizing effect via destructive autophagy. ACS Biomater Sci Eng. 2019;5(3):1569-1579. doi:10.1021/acsbiomaterials.8b01181

11. Xing C, Xie Z, Liang Z, et al. 2D nonlayered selenium nanosheets: facile synthesis, photoluminescence, and ultrafast photonics. Adv Opt Mater. 2017;5(24):1700884. doi:10.1002/adom.201700884

12. Tang X, Liang W, Zhao J, et al. Fluorinated phosphorene: electrochemical synthesis, atomistic fluorination, and enhanced stability. Small. 2017;13(47):1702739. doi:10.1002/smll.v13.47

13. Fan T, Xie Z, Huang W, Li Z, Zhang H. Two-dimensional nonlayered selenium nanoflakes: facile fabrications and applications for self-powered photo-detector. Nanotechnology. 2019;30(11):114002. doi:10.1088/1361-6528/ab0ed0

14. Gong L, Yan L, Zhou R, Xie J, Wu W, Gu Z. Two-dimensional transition metal dichalcogenide nanomaterials for combination cancer therapy. $J$ Mater Chem B. 2017;5(10):1873-1895. doi:10.1039/C7TB00195A

15. Song G, Cheng L, Chao Y, Yang K, Liu Z. Emerging nanotechnology and advanced materials for cancer radiation therapy. Adv Mater. 2017;29(32):1700996. doi:10.1002/adma.201700681

16. Shen S, Chao Y, Dong Z, et al. Bottom-up preparation of uniform ultrathin rhenium disulfide nanosheets for image-guided photothermal radiotherapy. Adv Funct Mater. 2017;27(28):1700250. doi:10.1002/adfm.v27.28

17. Qi F, Liu R. Tumor-targeted and biocompatible $\mathrm{MoSe}_{2}$ nanodots@ albumin nanospheres as a dual-modality therapy agent for synergistic photothermal radiotherapy. Nanoscale Res Lett. 2019;14(1):67. doi:10.1186/s11671-019-2896-z

18. Cheng L, Yuan C, Shen S, et al. Bottom-up synthesis of metal-iondoped $\mathrm{WS}_{2}$ nanoflakes for cancer theranostics. ACS Nano. 2015;9 (11):11090-11101. doi:10.1021/acsnano.5b04606

19. Chen J, Li X, Liu X, et al. Hybrid $\mathrm{MoSe}_{2}$-indocyanine green nanosheets as a highly efficient phototheranostic agent for photoacoustic imaging guided photothermal cancer therapy. Biomater Sci. 2018;6(6):1503-1516. doi:10.1039/c8bm00104a

20. Lin J, Wang S, Huang P, et al. Photosensitizer-loaded gold vesicles with strong plasmonic coupling effect for imaging-guided photothermal/photodynamic therapy. ACS Nano. 2013;7(6):5320-5329. doi:10.1021/nn4011686
21. El-Sayed IH, Huang X, El-Sayed MA. Selective laser photo-thermal therapy of epithelial carcinoma using anti-EGFR antibody conjugated gold nanoparticles. Cancer Lett. 2006;239(1):129-135. doi:10.1016/j. canlet.2005.07.035

22. Lee C, Lim K, Kim SS, et al. Near infrared light-responsive heatemitting hemoglobin hydrogels for photothermal cancer therapy. Colloids Surf B Biointerfaces. 2019;176:156-166. doi:10.1016/j. colsurfb.2018.12.070

23. Chen J, Liu H, Zhao C, et al. One-step reduction and PEGylation of graphene oxide for photothermally controlled drug delivery. Biomaterials. 2014;35(18):4986-4995. doi:10.1016/j.biomaterials.2014.02.032

24. Ocsoy I, Isiklan N, Cansiz S, Özdemir N, Tan W. ICG-conjugated magnetic graphene oxide for dual photothermal and photodynamic therapy. RSC Adv. 2016;6(36):30285-30292. doi:10.1039/C6RA06798K

25. Choudhury KR, Lee J, Chopra N, et al. Highly efficient hole injection using polymeric anode materials for small-molecule organic lightemitting diodes. Adv Funct Mater. 2009;19(3):491-496.

26. Chen H, Liu T, Su Z, Shang L, Wei G. 2D transition metal dichalcogenide nanosheets for photo/thermo-based tumor imaging and therapy. Nanoscale Horiz. 2018;3(2):74-89. doi:10.1039/C7NH00158D

27. Ma Y, Liu X, Ma Q, Liu Y. Near-infrared nanoparticles based on indocyanine green-conjugated albumin: a versatile platform for imaging-guided synergistic tumor chemo-phototherapy with temperatureresponsive drug release. Onco Targets Ther. 2018;11:8517. doi:10.2147/OTT.S183887

28. Abbas M, Zou Q, Li S, Yan X. Self-assembled peptide-and protein-based nanomaterials for antitumor photodynamic and photothermal therapy. $A d v$ Mater. 2017;29(12):1605021. doi:10.1002/adma.201700681

29. Xie Z, Wang D, Fan T, et al. Black phosphorus analogue tin sulfide nanosheets: synthesis and application as near-infrared photothermal agents and drug delivery platforms for cancer therapy. J Mater Chem B. 2018;6(29):4747-4755.

30. Luo M, Fan T, Zhou Y, Zhang H, Mei L. 2D black phosphorus-based biomedical applications. Adv Funct Mater. 2019;29(13):1808306.

31. Fan T, Zhou Y, Qiu M, Zhang H. Black phosphorus: a novel nanoplatform with potential in the field of bio-photonic nanomedicine. $J$ Innov Opt Health Sci. 2018;11(6):1830003.

32. Fang J, Nakamura H, Maeda $H$. The EPR effect: unique features of tumor blood vessels for drug delivery, factors involved, and limitations and augmentation of the effect. Adv Drug Deliv Rev. 2011;63(3):136-151.

33. Grimes DR, Kannan P, McIntyre A, et al. The role of oxygen in avascular tumor growth. PLoS One. 2016;11(4):e0153692.

34. Qian X, Shen S, Liu T, Cheng L, Liu Z. Two-dimensional $\mathrm{TiS}_{2}$ nanosheets for in vivo photoacoustic imaging and photothermal cancer therapy. Nanoscale. 2015;7(14):6380-6387.

35. Tan C, Lai Z, Zhang H. Ultrathin two-dimensional multinary layered metal chalcogenide nanomaterials. Adv Mater. 2017;29(37):1701392.

36. Chen Y, Wang L, Shi J. Two-dimensional non-carbonaceous materials-enabled efficient photothermal cancer therapy. Nano Today. 2016;11(3):292-308

37. Dhenadhayalan N, Sriram MI, Lin KC. Aptamer-based fluorogenic sensing of interferon-gamma probed with $\mathrm{ReS}_{2}$ and $\mathrm{TiS}_{2}$ nanosheets. Sens Actuators B Chem. 2018;258:929-936.

38. Tao W, Ji X, Xu X, et al. Antimonene quantum dots: synthesis and application as near-infrared photothermal agents for effective cancer therapy. Angew Chem Int Ed Engl. 2017;56(39):11896-11900.

39. Sun Z, Xie H, Tang S, et al. Ultrasmall black phosphorus quantum dots: synthesis and use as photothermal agents. Angew Chem Int Ed Engl. 2015;54(39):11526-11530.

40. Qiu M, Wang D, Liang W, et al. Novel concept of the smart NIRlight-controlled drug release of black phosphorus nanostructure for cancer therapy. Proc Natl Acad Sci U S A. 2018;115(3):501-506.

41. Zheng Y, Hunting DJ, Ayotte P, Sanche L. Radiosensitization of DNA by gold nanoparticles irradiated with high-energy electrons. Radiat Res. 2008;169(1):19-27. 
42. Hossain M, Su M. Nanoparticle location and material-dependent dose enhancement in X-ray radiation therapy. J Phys Chem A. 2012;116 (43):23047-23052.

43. Ashton JR, Castle KD, Qi Y, Kirsch DG, West JL, Badea CT. Dualenergy CT imaging of tumor liposome delivery after gold nanoparticle-augmented radiation therapy. Theranostics. 2018;8(7):1782.

44. Gao S, Cheng X, Li J. Lipid nanobubbles as an ultrasound-triggered artesunate delivery system for imaging-guided, tumor-targeted chemotherapy. Onco Targets Ther. 2019;12:1841.

45. Park DH, Cho J, Kwon OJ, Yun CO, Choy JH. Biodegradable inorganic nanovector: passive versus active tumor targeting in siRNA transportation. Angew Chem Int Ed Engl. 2016;55(14):4582-4586.
46. Fang C, Shi B, Pei YY, Hong MH, Wu J, Chen HZ. In vivo tumor targeting of tumor necrosis factor- $\alpha$-loaded stealth nanoparticles: effect of MePEG molecular weight and particle size. Eur J Pharm Sci. 2006;27(1):27-36.

47. Perry JL, Reuter KG, Kai MP, et al. PEGylated PRINT nanoparticles: the impact of PEG density on protein binding, macrophage association, biodistribution, and pharmacokinetics. Nano Lett. 2012;12 (10):5304-5310. 


\section{Supplementary materials}
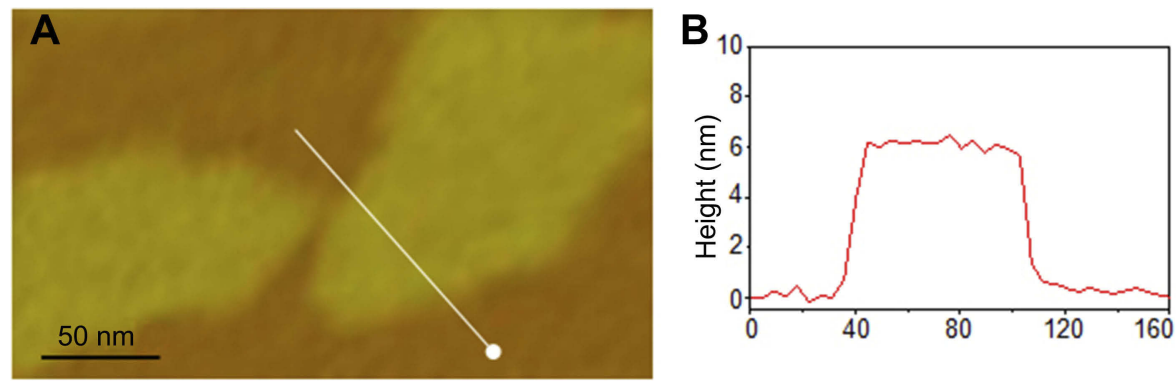

Figure SI (A) The AFM image and (B) height analysis of $\mathrm{TiS}_{2}$ nanosheets.

Abbreviations: AFM, atomic force microscopy; $\mathrm{TiS}_{2}$, titanium disulfide.

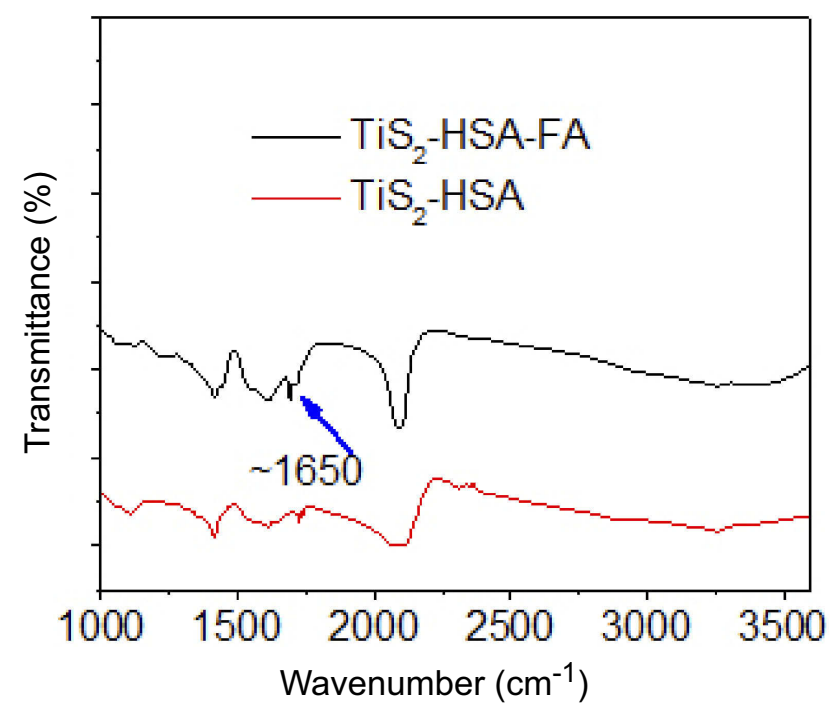

Figure S2 The FT-IR spectrum of $\mathrm{TiS}_{2}-\mathrm{HSA}$ and $\mathrm{TiS}_{2}-\mathrm{HSA}-\mathrm{FA}$.

Abbreviations: $\mathrm{TiS}_{2}$, titanium disulfide; HSA, human serum albumin; FA, folic acid.

\section{Publish your work in this journal}

OncoTargets and Therapy is an international, peer-reviewed, open access journal focusing on the pathological basis of all cancers, potential targets for therapy and treatment protocols employed to improve the management of cancer patients. The journal also focuses on the impact of management programs and new therapeutic agents and protocols on patient perspectives such as quality of life, adherence and satisfaction. The manuscript management system is completely online and includes a very quick and fair peer-review system, which is all easy to use. Visit http://www.dovepress.com/ testimonials.php to read real quotes from published authors. 